\title{
Populism within Europe and beyond its borders. Eine Expertenkonferenz der Konrad-Adenauer-Stiftung am 16./17. Dezember 2012 in Brüssel
}

Populismus ist ein vielschichtiges Phänomen mit praktischer Relevanz, das nicht nur in Europa seinen festen Platz gefunden hat. Zu diesem eindeutigen Befund kam eine Expertenkonferenz, organisiert vom Multinationalen Entwicklungsdialog der Konrad-AdenauerStiftung, die am 16. und 17. Dezember 2012 in Brüssel stattfand. Das Konzept der Tagung hatten Andrea Ostheimer de Sosa, Programmdirektorin des Multinationalen Entwicklungsdialogs, und der Populismusforscher Florian Hartleb entwickelt. In Impulsreferaten und Diskussionen äußerten sich Experten zu den Weltregionen. Vorbild war die erste wissenschaftliche Tagung 1967 an der London School of Economics (LSE), die genau diesen globalen Ansatz verfolgte, der danach jedoch kaum mehr aufgegriffen wurde.

Am Beginn der Tagung stand der Versuch, Populismus konzeptionell zu bestimmen. Florian Hartleb als Moderator machte deutlich, dass Populismus ein schwer zu fassender Begriff ist. Hans-Jürgen Puble (Universität Frankfurt am Main) erläuterte Kontinuitäten und Wandel von Populismen. Er hatte schon 1983 einen Aufsatz zur immer noch aktuellen und diskutierten Frage „Was ist Populismus?“ veröffentlicht. Immer wieder gebe es populistische Wellen in Modernisierungsprozessen. Guy Hermet (Universität Paris) konstatierte, dass Populismus immer wieder als Schimpfwort im politischen Tagesgeschäft gebraucht werde. Cristóbal Rovira Kaltwasser (Universität Sussex) bezeichnete Populismus als „dünne Ideologie“, die nicht einfach mit Klientelismus, charismatischer Führung oder Neofaschismus gleichzusetzen sei.

Das nächste Panel behandelte Europa, mit Fallstudien zu den Niederlanden und Dänemark, zu Griechenland und zur Slowakei. Moderator Florian Hartleb betonte, dass Populismus nicht erst seit der globalen Finanzkrise zum Dauerbrenner der europäischen Politik geworden sei. Paul Lucardie (Universität Groningen) behandelte den Fall der Niederlande, wo vor allem Geert Wilders mit einem radikalen Antiislamismus für Diskussionen sorgt. Feste Tradition hat der Populismus in Dänemark, wo er "wohlfahrtschauvinistische Motive“ der Wähler bedient und entsprechende Ressentiments schürt. Andreas Klein (Konrad-Adenauer-Stiftung Hamburg) strich auch die Kampagnenfähigkeit derartiger Parteien heraus. Im krisengeschüttelten Griechenland ist ebenso eine Reihe von rechten und linken Populismen zu beobachten wie in der Slowakei. Als landeskundige Referenten wirkten hier Manos G. Papazoglou (Universität Peleponnes) sowie Olga Gyárfášsvá (Comenius Universität Bratislava). Karin Priester (Universität Münster) stellte als Kommentatorin heraus, dass Populismus insbesondere in Griechenland eine enge Verbindung zum Klientelismus aufweise.

Die folgenden Panels gingen über Europas Grenzen hinaus und betrachteten auch die klientelistischen Züge als Begleiterscheinungen von Populismus. Frank Priess, stellvertretender Leiter der Hauptabteilung Europäische und Internationale Zusammenarbeit der Konrad-Adenauer-Stiftung und Kenner Lateinamerikas, führte in die „Americas“ ein. In letzter Zeit hat es eine Debatte um den bewegungsförmigen, fundamentalistischen Populismus der Tea Party gegeben. Aus diesem Grund widmeten sich gleich zwei Referenten, Joseph Lowndes (Universität Oregon) und Alexander Kühne (Universität Halle-Wittenberg) aus der USamerikanischen beziehungsweise europäisch-deutschen Perspektive diesem Thema und zeigten, dass Populismus in den USA eine eigene Traditionslinie aufweist. Das gilt auch für 
Argentinien: Nikolaus Werz (Universität Rostock) erinnerte daran, dass der Peronismus 2013 seinen 70. Jahrestag begeht. Gerade dessen ökonomische Leistungsbilanz wird immer wieder kritisiert. Anthony Pereira (King's College London) sprach über den brasilianischen Präsidenten Lula da Silva, seine besondere Beziehung zu den Massen, und über illiberale Tendenzen im Lande. Arnold Kammel (Politische Akademie Österreich) strich als Kommentator die sozialen Ungleichheiten als Nährboden für Populismus heraus.

Aurel Croissant (Universität Heidelberg) leitete anschließend das Panel zu Asien und führte in die Thematik ein. Mit der Jahrtausendwende gelangte der Begriff - angesichts einiger signifikanter empirischer Fälle - in die asiatische Hemisphäre. Speziell in Thailand, Malaysia, Südkorea, Indonesien, den Philippinen und Japan fand Populismus begrifflich (neue) Anwendung und bietet Anlass zur komparativen Forschung. Ein besonders eindeutiger, für Komparatistik geeigneter Fall findet sich in Thailand mit der Thaksin-Familie. Peter Warr (Australian National University) analysierte diesen Fall. Während der frühere Premierminister Thaksin Shinawatra als Justizflüchtling im Exil lebt, regiert mittlerweile seine Schwester, die den Kult nach wie vor hochhält. Der Thaksin-Populismus ist anti-intellektuell, gegen das Zentrum (hier Bangkok) gerichtet und anti-elitär. In den Philippinen gibt es nicht diesen offensichtlichen Populismus wie in Thailand. Das machte Eva-Lotta Hedman (London School of Economics) deutlich. Kosuke Mizono (Kyoto-Universität) stellte den indonesischen Fall da, wo die Eliten selbst korrumpiert seien. Haruko Sato (Osaka Universität) zeigte auf, dass mit der letzten Wahl in Japan Populismus auf dem Vormarsch sei. Führung mit starker Hand und eine Identitätspolitik erinnern an den gegenwärtigen Rechtspopulismus in Europa. Kommentator William Case (Südostasienforschungszentrum der City University in Hongkong) sprach sich dafür aus, den Populismusbegriff nicht zu stark einzugrenzen. Bisher gebe es in Asien nur einen klaren Fall: Thailand.

Als letzte, häufig vernachlässigte Weltregion stand Subsahara-Afrika auf dem Programm. Andrea Ostheimer de Sosa führte in Region und bisherige Forschung ein. In Äthiopien etwa gehe es sehr stark um die Frage der Landreformen, in Südafrika, so Siegmar Schmidt (Universität Landau), zudem insbesondere um den Befreiungsmythos. In Äthiopien gibt es, Stefan Brüne (Universität Hamburg) zufolge, autokratische Herrschaftsstrukturen und einen Populismus „von oben“, die einen Vergleich zum „kompetitiven Autoritarismus“ lateinamerikanischer Prägung erkenntnisreich machen könnten. Beatrice Schlee (Universität Freiburg) und Goodhope Ruswa (Universität Zimbabwe) stellten mit Blick auf Zimbabwe erste gemeinsame Forschungsergebnisse vor. Populistische Strategien, etwa im Zusammenhang mit der Frage der Landreformen, gedeihen hier in den paternalistischen Strukturen. Zuletzt versuchte Florian Hartleb die Ergebnisse der Tagung zusammenzuführen. Immer falle die antagonistische Natur von Populismus ins Auge: „Wir gegen die-da-oben“, „wir gegen die-dadraußen“, Populismus „von unten“ und „von oben“ sowie der Gegensatz zwischen Zentrum und Peripherie. 